\title{
Diagnostic Accuracy of Contrast-enhanced Ultrasonography Liver Imaging Reporting and Data System in Hepatocellular Carcinoma: A Systematic Review
}

\author{
HuiFang Li \\ Sun Yat-sen University Cancer Center \\ Wei Huang \\ Sun Yat-sen University Cancer Center \\ Wei Zheng \\ Sun Yat-sen University Cancer Center \\ Qing Li \\ Sun Yat-sen University Cancer Center \\ YunZhu Dai \\ Sun Yat-sen University Cancer Center

\section{Lie Zheng} \\ Sun Yat-sen University Cancer Center \\ RuHai Zou ( $\square$ zourh@sysucc.org.cn ) \\ Sun Yat-sen University Cancer Center
}

\section{Research Article}

Keywords: contrast-enhanced ultrasonography, hepatocellular carcinoma, diagnosis, Liver Imaging Reporting and Data System (LI-RADS), sensitivity

Posted Date: April 8th, 2021

DOI: https://doi.org/10.21203/rs.3.rs-379866/v1

License: @ (i) This work is licensed under a Creative Commons Attribution 4.0 International License. Read Full License 


\section{Abstract}

Background: Contrast-enhanced Ultrasonography Liver Imaging Reporting and Data System (CEUS LI-RADS) released by American College of Radiology was a widely used reporting system for patients at risk with hepatocellular carcinoma (HCC). In CEUS LI-RADS, the categories range from definitely benign (LR-1), probably begin (LR-2), intermediate probability of malignancy (LR-3), probably HCC (LR-4) to definitely HCC (LR-5), malignancy (LR-M), or definite tumor in vein (LR-TIV).

Methods: We searched MEDLINE, Web of Science, Cochrane, Embase, and Chinese databases to obtain eligible studies reporting on the diagnostic performance of CEUS LI-RADS in patients at risk for HCC.

Results: Twelve studies were eligible in the analysis, including 5275 patients, 5739 observations, and 4066 HCCs. The pooled sensitivity and specificity were $70 \%$ (95\% Confidence Interval [Cl] 65\%-74\%), 94\% (95\% Cl, 91\%-96\%) of LR-5 category as predictors of HCC, respectively. The pooled sensitivity and specificity of LR-M category as a predictor of non-HCC malignancy were $83 \%(95 \% \mathrm{Cl}, 71 \%-91 \%), 94 \%$ (95\% Cl 88\%-97\%), respectively. The pooled proportions of HCCs were $1 \%(95 \% \mathrm{Cl} 0 \%-6 \%$ ) for LR-2, 20\% (95\% Cl, 9\%-34\%) for LR-3, 78\% (95\% Cl, 67\%-88\%) for LR-4, 97\% (95\% Cl, 94\%-99\%) for LR-5, 40\% (95\% Cl, 23\%-58\%) for LR-M and $100 \%(95 \% \mathrm{Cl}, 93 \%-100 \%)$ for LR-TIV.

Conclusion: CEUS LI-RADS is an important tool for the diagnosis of HCC.

\section{Introduction}

Hepatocellular carcinoma (HCC) was accounted for the majority of primary hepatic neoplasm, threated to human health severely, and ranked fourth for cancer death in 2018[1]. For those who had liver cirrhosis, HCC is the main cause of death. The prognosis of advanced liver cancer is relatively poor with a low 5-year survival rate $(\mathbb{2} 20 \%)[2]$. If diagnosed early, the prognosis could be greatly improved (5-year survival $₫ 50 \%)[3,4]$.

Imaging plays an important role in the early diagnosis of HCC. It is well recommended that histological biopsy is not necessary when the typical features of HCC are showed by appropriate imaging examination in patients at high risk[5]. Contrast-enhanced ultrasound (CEUS) has been routinely used to distinguish benign and malignant liver nodules in the clinic and provides useful diagnostic information[6, 7]. The American College of Radiology has developed the CEUS Liver Imaging Reporting and Data System (CEUS LI-RADS), which is a diagnostic algorithm aimed to standardize interpreting imaging results of the liver lesions at risk of HCC and can help clinicians decide whether to proceed with the next more aggressive examination[8, 9]. The initial version of CEUS LI-RADS was launched in 2016 and updated in 2017. According to the algorithm, liver nodules in patients at high risk for HCC are classified into five major categories (from LR-1 to LR-5), reflecting the probability of HCC. Additionally, the LR-M category refers to probably or definitely malignant, but not necessarily HCC.

Many studies have shown CEUS LI-RADS was of high sensitivity and specificity in diagnosing HCC[10]. However, some were with relatively small samples because the algorithm has been developed for a short time compared with LI-RADS in CT/MRI. The diagnostic performance of CEUS LI-RADS and the percentage of $\mathrm{HCC}$ and overall malignancy in each category have not been thoroughly evaluated. Thus, we carry out a meta-analysis to investigate the diagnostic performance of the LR-5 category for HCC, LR-M category as a predictor of non-HCC malignancy, and pooled proportion of HCC and overall malignancy for each CEUS LI-RADS category.

\section{Methods}

This study followed the Preferred Reporting Items for Systematic Reviews and Meta-Analyses (PRISMA) guideline for conduct and reporting. It used analytical methods currently recommended by the Diagnostic Test Accuracy Working Group of the Cochrane Collaboration and the Agency for Healthcare Research and Quality. The protocol was registered in the PROSPERO (CRD 42020175194)

\section{Search Strategy}

We systematically searched the MEDLINE, Embase, Web of Science, Cochrane CENTRAL, Scopus databases, and Chinese database from August 2016 to April 2020, with no language restrictions. The literature search strategy was following specific keywords and Boolean operators. The keywords were including: "CEUS or contrast-enhanced ultrasound," "LI-RADS," "HCC or Hepatocellular carcinoma." The detailed search terms were listed in Appendix 1.

\section{Selection Criteria}

Studies were reviewed with respect to eligibility: (a) Patients at high risk of HCC presented with untreated liver nodules; (b) Index test, CEUS; (c) LI-RADS version 2016 (v.2016) or v.2017 were used to evaluate lesions; (d) reference standard based on pathological diagnosis or clinical follow-up (e) original research including prospective or retrospective studies; (f) True positive, false positive, true negative and false negative of the LR-5 category for HCC; ( $g$ ) the proportions of HCCs and non-HCC malignancies in each LI-RADS category. Studies were excluded if they: (a) liver observations that were treated before the index test (CEUS); (b) insufficient data were required to calculate sensitivity and specificity. (c) the possibility of duplicate data. Initially, two reviewers (H.F.L and R.H.Z) independently screened abstracts and titles. Then the potentially relevant articles were accessed by full-text review according to selection criteria. Any disagreements were resolved through discussion with a third reviewer (W.H).

\section{Data Extraction}

Available data extracted included (1) study characteristics (study design, publication year, journal, country where the study was performed, etc.); (2) patient characteristics (number, age and gender); (3) liver observations characteristics (quantities, size range, chronic liver disease); (4) Type of US probe and type of contrast agents; (5) Accuracy data for CEUS findings: the numbers of true positives, false positives, false negatives, and true negatives; (6) image review

Page 2/10 
method; (7) reference standards. Two reviewers (H.F.L and R.H.Z) independently extracted data using a standardized form. Then disagreements were resolved through discussion by consensus or a third reviewer (W.H).

\section{Assessment Of The Quality Of The Included Studies}

The quality of the included studies was assessed according to the Quality Assessment of Diagnostic Accuracy Studies (QUADAS-2) tool which included the risk of bias for four domains (patient selection, index test, reference standard, and flow and timing) and the clinical applicability for the first three domains of the study characteristics[11]. Two reviewers (H.F.L. and R.H.Z, all had enough experience in radiology) independently used the QUADAS-2 tool to assess the quality. The reviewers did not blindly review these articles. Discrepancies were resolved through discussion with a third reviewer (W.H).

\section{Data Synthesis And Statistical Analysis}

To estimate summary sensitivities and specificities of LR- 5 and LR-M and their $95 \%$ confidence intervals (95\% Cls), a meta-analysis was performed using a random-effects model. To present the variation between studies in sensitivity and specificity, study results were showed in forest plots, and receiver operating characteristic (ROC) plots with a 95\% estimate region.

To determine the pooled proportions of HCCs and overall malignancies for each LI-RADS category, a random-effects model was applied, with forest plots and $95 \%$ Cls. Study heterogeneity in each LI-RADS category was evaluated by the Higgins I 2 statistic, which exceeds $50 \%$ regarded as possible substantial heterogeneity.

Heterogeneity was quantified with the $\mathrm{Q}$ test $(\mathrm{P}<0.05$, indicating significant heterogeneity). We conducted meta-regression analyses to explore potential covariates of heterogeneity based on the study characteristics. Such covariates were as following: (1) study type and design (case-control, cohort study, prospective or retrospective); (2) patients enrollment (consecutive versus selective); (3) CEUS LI-RADS version (v.2017 versus v.2016); (4) image reviewers (multiple independent reviewers versus single reviewers); and (5) reference standard (pathology only versus pathology or clinal follow-up).

To assess publication bias, we performed funnel plots and the Egger's test.

Statistical analyses were done using R version 3.3.2 (The R Foundation for Statistical Computing, Vienna, Austria) and STATA software (Version 12.0; Stata Corporation).

\section{Results}

\section{Literature Search}

Through our electronic search, 406 studies were initially identified, and 70 of these were duplicates. Fifty-one studies were relevant after reading titles and abstracts. After scrutinizing the full text, 12 articles[12-16, 10,17-22] with sufficient detail that meet our research design were included (Fig. 1). Details of the excluded studies are showed in Appendix 1, See Fig. 1 for the study selection process.

\section{Characteristics of The Included Studies}

The important characteristics of the finally included studies are listed in Table 1. A total of 5275 patients with 5739 lesions were evaluated in this article (Table 2). All studies were full-text publications that contain enough data and details to analyze. All included studies except for one[20] had been carried out in a single center. Most of these studies had a retrospective design, only two prospective studies. And in these retrospective studies, 1 study[16] was a casecontrol design. Liver nodules were analyzed according to LI-RADS v.2017 in 9 studies[14, 12, 16, 10, 22, 17, 21, 15, 19], and v.2016 in the other 3 articles[18, 20, 13].

The studies mostly were conducted in Asia, in which China and South Korea account for the majority; 1 in Italy[20], 1 in German[18]. In 3 of the 12 available studies, the publication was English, and the rest was Chinese[13, 12, 14]. 
Table 1

Characteristic of Included Studies

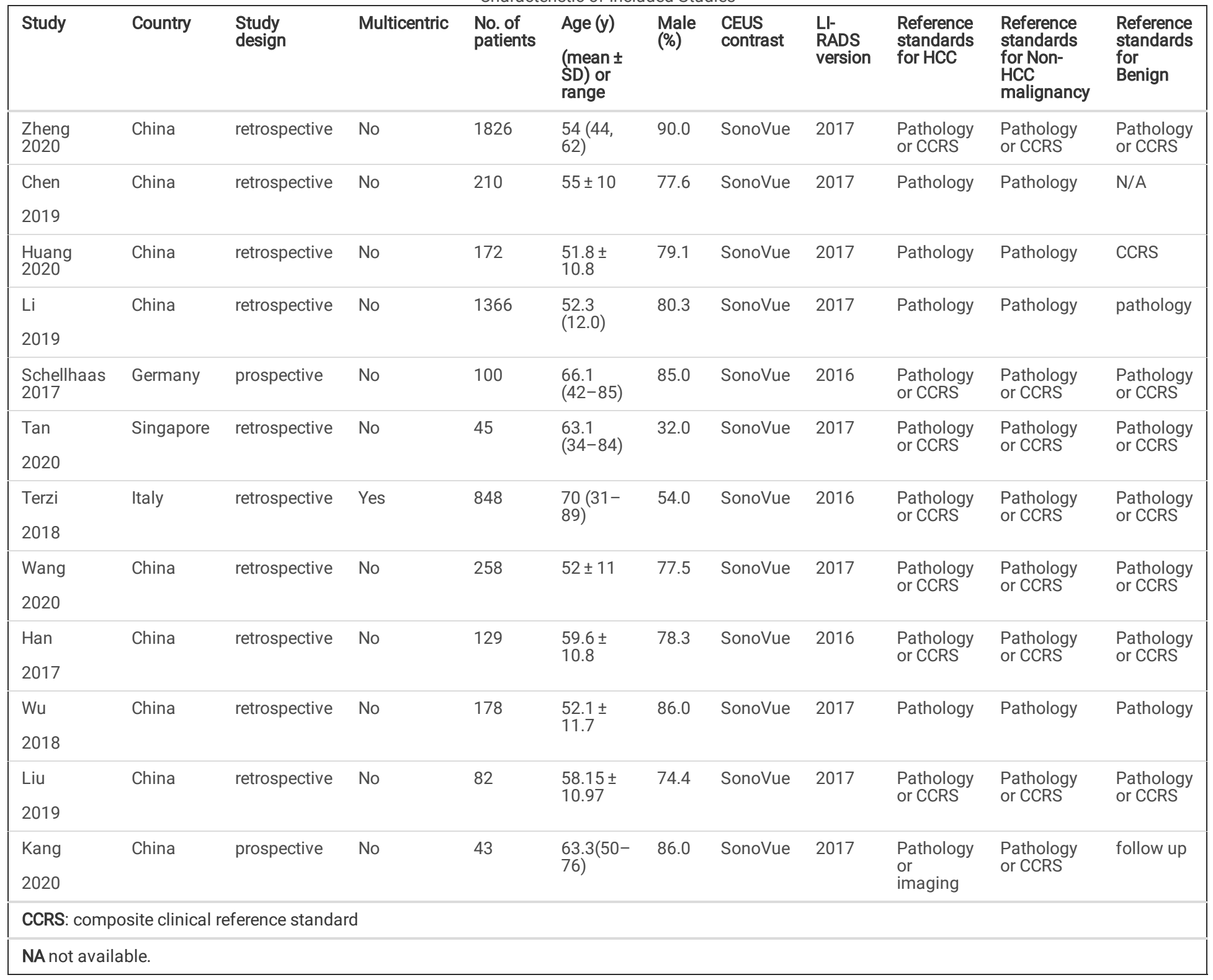


Table 2

Number of HCC and Malignancy Observations per LI-RADS Category per Study

\begin{tabular}{|c|c|c|c|c|c|}
\hline Study & $\begin{array}{l}\text { Observations } \\
\mathrm{n}\end{array}$ & Observations size (mm) & $\begin{array}{l}\mathrm{HCC} \\
\mathrm{n}\end{array}$ & Non-HCC malignancy, n & Benign, $\mathbf{n}$ \\
\hline Zheng 2020 & 2020 & N/A & 1514 & 138 & 368 \\
\hline Chen 2018 & 210 & N/A & 105 & 105 & 0 \\
\hline Huang 2020 & 175 & $16.1 \pm 3.4$ & 105 & 3 & 67 \\
\hline Li 2019 & 1366 & $47(35)$ & 985 & 139 & 242 \\
\hline Schellhaas2017 & 100 & $52.2 \pm 78.9$ & 87 & 6 & 7 \\
\hline Tan 2020 & 46 & N/A & 37 & 9 & 0 \\
\hline Terzi 2018 & 1,006 & $20(5-150)$ & 820 & 53 & 133 \\
\hline Wang 2020 & 355 & 25 & 115 & 5 & 235 \\
\hline Han 2017 & 148 & N/A & 68 & 36 & 44 \\
\hline Wu 2018 & 197 & $29 \pm 16$ & 170 & 17 & 10 \\
\hline Liu 2019 & 82 & $37.04 \pm 30.69$ & 48 & 1 & 33 \\
\hline Kang 2020 & 34 & $21(20-35)$ & 12 & 1 & 21 \\
\hline
\end{tabular}

\section{Methodological Quality}

Our evaluation of study quality with the QUADAS-2 tool is reported in Appendix 2. Overall, these included studies were considered to be at high risk of bias and high concerns about applicability. Only one study presented low bias in all four domains and applicability concerns[15]. The major risk of bias and applicability concerns were related to patient selection, reference standard. 2 articles[16, 22] selectively enrolled study subjects $(1[16]$ was case-control). This was considered high risk because it may affect the prevalence of HCC in the study by the authors.

Regarding the index test, 1 study used CEUS and the CT/MRI[22], which may be a source of bias. The reference standard was a potential risk of bias because these articles used different reference standards to diagnose liver nodes. For example, only using pathology would be appropriate to exclude some patients[23]. Most studies did not report whether or not the assessment of the index test was blinded to the reference standard. On the other hand, the Flow and Timing domain in most articles was not reported, so the risk of bias was unclear.

\section{Diagnostic Performance Of Ceus Lirads}

The overall per-lesion sensitivity and specificity of CEUS LR-5 for the diagnosis of HCCs from 12 studies were $70 \%$ (95\% Cl, $65 \%-74 \%)$ and $94 \%$ (95\% Cl, 91\%-96\%), respectively (Appendix 3). The pooled estimate of diagnostic odds ratio (DOR) was 61.12 (95\% Cl 34.81-107.32). The summary of ROC is provided in Appendix 5.

The overall per-lesion sensitivity and specificity of LR-M as a predictor of non- $\mathrm{HCC}$ malignancy from 10 studies were $83 \%(95 \% \mathrm{Cl}, 71 \%-91 \%)$ and $94 \%$ (95\% $\mathrm{Cl}$, 88\%-97\%), respectively (Appendix 4).

\section{Meta-regression Analysis}

We explored potential heterogeneity using the nation of publication, study design, subject enrollment, LI-RADS version, imaging reviewer, and reference standard as covariates. For LI-RADS category 5, all these factors, except for imaging reviewers, significantly influenced sensitivities or specificities. And for LIRADS M, only reference standard had a significant impact on the pooled sensitivity (Pख0.01). The result was summarized in Table 3 and Table 4.

\section{Proportions of HCC and overall malignancies for each LI-RADS category}

The forest plot data of the percentages of HCC and overall malignancies for each LI-RADS category in each study are provided in Appendix 6. The pooled percentages of $\mathrm{HCC}$ and overall malignancy, respectively, were $0 \%(95 \% \mathrm{Cl}, 0-2 \%)$ and $0 \%(0-2 \%)$ for LR-1, 1\% (95\% Cl 0-6\%) and 1\% (0-6\%) for LR-2, 20\% (9-34\%) and 23\% (10-37\%) for LR-3, 78\% (67-88\%) and 82\% (72-91\%) for LR-4, 97\% (94-99\%) and 99\% (98-100\%) for LR-5, 40\% (23-58\%) and 97\% (8799\%) for LR-M, and 100\% (93-100\%) and 100\% (93-100\%) for LR-TIV (Fig. 2). Meta-regression was performed for each category (Appendix 7).

\section{Publication Bias}

The linear regression test of funnel plot asymmetry indicated no significant publication bias $(P=0.50)$. 
Table 3

Results of meta-regression analysis of the sensitivity and specificity of LI-RADS category 5 for diagnosing HCC

\begin{tabular}{|c|c|c|c|c|}
\hline & Sensitivity & $P$ value & Specificity & $P$ value \\
\hline Nation of publication & & $\varangle 0.01$ & & $₫ 0.01$ \\
\hline $\operatorname{Asian}(n=10)$ & $71(67-75)$ & & $95(92-98)$ & \\
\hline Non-Asian(n = 2) & $66(57-74) \otimes$ & & $91(81-100)$ & \\
\hline Study design & & 0.04 & & 0.03 \\
\hline Prospective study $(\mathrm{n}=2)$ & $59(44-75)$ & & $86(70-100)$ & \\
\hline Retrospective study $(n=10)$ & $70(66-75)$ & & $95(92-97)$ & \\
\hline Subject enrollment & & $\varangle 0.01$ & & 0.63 \\
\hline Consecutive $(n=10)$ & $72(68-75)$ & & $94(91-97)$ & \\
\hline Selective $(\mathrm{n}=2)$ & $53(40-66)$ & & $96(92-100)$ & \\
\hline LI-RADS version & & 0.01 & & 0.08 \\
\hline $2017(n=9)$ & $72(69-76)$ & & $95(92-98)$ & \\
\hline $2016(n=3)$ & $64(58-70)$ & & $94(88-100)$ & \\
\hline Imaging reviewer & & 0.28 & & 0.33 \\
\hline Inexperienced(n = 1) & $76(63-88)$ & & $94(87-100)$ & \\
\hline experienced $(n=11)$ & $69(65-73)$ & & $94(92-97)$ & \\
\hline Reference standard & & 0.02 & & $\bowtie 0.01$ \\
\hline Pathology $(n=2)$ & $75(67-82)$ & & $86(83-90)$ & \\
\hline Pathology or CCRS $(n=10)$ & $68(64-73)$ & & $96(95-97)$ & \\
\hline
\end{tabular}

Table 4

Results of meta-regression analysis of the sensitivity and specificity of LI-RADS category M for diagnosing HCC

\begin{tabular}{|c|c|c|c|c|}
\hline & Sensitivity & $P$ value & Specificity & $P$ value \\
\hline Nation of publication & & 0.16 & & 0.72 \\
\hline $\operatorname{Asian}(\mathrm{n}=8)$ & $86(77-95)$ & & $92(87-98)$ & \\
\hline Non-Asian $(n=2)$ & $73(47-98)$ & & $97(92-100)$ & \\
\hline Study design & & 0.18 & & 0.08 \\
\hline Prospective study $(\mathrm{n}=2)$ & $59(13-100)$ & & $98(95-100)$ & \\
\hline Retrospective study $(\mathrm{n}=8)$ & $86(78-93)$ & & $92(87-97)$ & \\
\hline Subject enrollment & & 0.11 & & 0.21 \\
\hline Consecutive $(n=8)$ & $82(73-91)$ & & $94(91-98)$ & \\
\hline Selective(n = 2) & $96(90-100)$ & & $87(70-100)$ & \\
\hline LI-RADS version & & 0.47 & & 0.93 \\
\hline $2017(n=7)$ & $81(65-98)$ & & $97(93-100)$ & \\
\hline $2016(n=3)$ & $83(70-95)$ & & $91(85-97)$ & \\
\hline Reference standard & & $\otimes 0.01$ & & 0.39 \\
\hline Pathology $(n=2)$ & $65(49-80)$ & & $91(80-100)$ & \\
\hline Pathology or CCRS(n= 8) & $87(82-93)$ & & $94(90-99)$ & \\
\hline
\end{tabular}


CEUS LI-RADS, released by the American College of Radiology in 2016 and updated in 2017, has been widely used and recognized by international scientific societies. However, the diagnostic performance of CEUS LI-RADS has not been widely evaluated and validated.

The findings showed a significant number of HCCs and malignant cases in the higher LI-RADS categories (LR-1 to LR-5). This pooled analysis found that the CEUS LI-RADS category LR-5 had a high specificity $(0.94$ [95\% $\mathrm{Cl} 0.91-0.96]$ for HCC. And the pooled sensitivity, specificity of LR-M, including ten studies as a predictor of non-HCC malignancy, were $70 \%$ and $94 \%$.

According to our systematic review, the pooled sensitivity and specificity of LR-5 were calculated, which were 0.70 ( $95 \% \mathrm{Cl} 0.65-0.74)$ and 0.94 ( $95 \% \mathrm{Cl}$ 0.91-0.96). To our knowledge, CEUS LR- 5 emphasizes high specificity close to $100 \%$ in diagnosing HCC in patients at risk for HCCs. Therefore, our result demonstrated that CEUS LI-RADS is an excellent system that can offer a good diagnostic value of HCC. Moreover, HCC guidelines also recommend CEUS as a diagnostic tool for HCC in expert centers[5].

Basing on current evidence, LR-M as a predictor of non-HCC malignancy showed a high pooled sensitivity $94 \%$ (95\% Cl, $88 \%-97 \%)$ but a relatively low specificity $83 \%(95 \% \mathrm{Cl}, 71 \%-91 \%)$ from 10 included studies. Notably, we found a high pooled proportion of HCCs (40\%) in the LR-M category, which means a low positive predictive value. It's necessary to modify the LR-M category to distinguish HCC from non-HCC malignancies[16].

We conducted a meta-regression analysis to explore the essential sources resulting in high heterogeneity. Finally, we observed considerable heterogeneity that mainly caused by the nation of publication, study design, and reference standard. It is worth noting that a statistically significant difference of specificity and sensitivity in the LR-5 category between the articles originating from Asia versus Non-Asian (Pख0.01). This may correct with the epidemiology of HCC, such as the etiology of the chronic liver disease and the presence of cirrhosis. Because HBV is endemic and cirrhosis is less common in Asia, unlike western countries' regions. Besides, our result shows that the pooled sensitivity and specificity of LI-RADS category 5 for diagnosing HCC in retrospective studies ( $\mathrm{n}=10$ ) are higher than the prospective study $(n=2)$. Because retrospective researches might induce some selection bias, another essential factor impacting the heterogeneity of results was that the use of different referent standards contributed to the heterogeneity (Pख0.05). Due to LI-RADS 1-3 corresponds to begin or intermediate malignancy probability, these patients are generally followed up. Thus two studies employed the only pathology as the reference standard, which will increase selection bias. Despite these limitations, our meta-analysis can still demonstrate the usefulness of CEUS LI-RADS.

In the present analysis, the results showed that pooled proportions of HCCs in 12 included studies were $0 \%$ for LR-1, $1 \%$ for LR-2, 20\% for LR-3, 78\% for LR-4, and $97 \%$ for LR-5. This result met the definition of LI-RADS, as the category increases, the degree of malignancy increased. According to the CEUS LI-RADS classification, LR-3 and LR-4 were defined as intermediate malignancy probability and the probability of HCC. However, the pooled proportions of HCC in LR-3 and LR-4 were high heterogeneity (Pख0.01). This could have been due to the inappropriate exclusion of some LR-3 and LR-4 lesions because of a lack of histology. The rate of HCC in class LR-3 also suggested that LR-3 observations should be considered a biopsy combination of patients' medical history. Moreover, we found the pooled proportion of malignancies was $97 \%$ for LR-M, consistent with the LR-M classification of malignancy not specific for HCC.

However, our meta-analysis had several crucial limitations. One limitation of this study is the poor reporting of primary studies. For example, the limited number of prospective research in this meta-analysis may introduce a bias toward increased diagnostic sensitivity. Moreover, the reference standard we used in this study were either histology or composite clinical reference standard. Some studies only used pathology as a referenced standard, and this may cause an inevitable selection bias because some enrolled patients may be excluded inappropriately. Also, we conducted meta-regression to explore the potential factors. Secondly, this meta-analysis is somewhat restricted by the date of the last search (2020.4). Therefore, it's unknown whether more high-quality researches published in the interim period. Thirdly, the number of included studies is small and may influence the outcome. Therefore, we did not conduct a subgroup to fully explore the possible sources of heterogeneity according to the characteristics of the article.

\section{Conclusions}

In conclusion, our meta-analysis reveals that CEUS LI-RADS is a useful tool for diagnosis HCC, and the specificity of LR-5 (94\%) is relatively high for diagnosing hepatocellular carcinoma. From LR-1 to LR-5, the possibility of malignancy increases. LR-M categories are highly suspicious for malignancy, but of a high proportion of $\mathrm{HCC}(40 \%)$.

\section{Abbreviations}

CEUS: Contrast-enhanced Ultrasonography

LI-RADS: Liver Imaging Reporting and Data System

HCC: Hepatocellular carcinoma

Cl: Confidence interval

PRISMA: Preferred Reporting Items for Systematic Reviews and Meta-Analyses

QUADAS-2: Quality Assessment of Diagnostic Accuracy Studies

ROC: Receiver operating characteristic

DOR: Diagnostic odds ratio 


\section{Declarations}

\section{Ethics approval and consent to participate}

Not applicable.

\section{Consent for publication}

Not applicable.

\section{Availability of data and materials}

The datasets used in the study are available from the corresponding author upon request.

\section{Competing interests}

Not applicable.

\section{Funding}

None.

\section{Authors' contributions}

Conception and design: HFL, WH, YZD, RHZ, LZ. Data collection: HFL, WH, RHZ. Quality assessment: HFL, WH, RHZ. Statistical analysis: HFL, WH, QL, YZD. Article writing: HFL, WZ, LZ. All authors have reviewed and approved the manuscript.

\section{Acknowledgements}

None

\section{References}

1. Bray F, Ferlay J, Soerjomataram I et al. (2018) Global cancer statistics 2018: GLOBOCAN estimates of incidence and mortality worldwide for 36 cancers in 185 countries. CA: a cancer journal for clinicians 68 (6):394-424. doi:10.3322/caac.21492

2. Jemal A, Ward EM, Johnson CJ et al. (2017) Annual Report to the Nation on the Status of Cancer, 1975-2014, Featuring Survival. JNCl: Journal of the National Cancer Institute 109 (9). doi:10.1093/jnci/djx030

3. Forner A, Llovet JM, Bruix J (2012) Hepatocellular carcinoma. Lancet 379 (9822):1245-1255. doi:10.1016/S0140-6736(11)61347-0

4. Forner A, Reig ME, de Lope CR, Bruix J (2010) Current strategy for staging and treatment: the BCLC update and future prospects. Semin Liver Dis 30 (1):61-74. doi:10.1055/s-0030-1247133

5. Marrero J, Kulik L, Sirlin C et al. (2018) Diagnosis, Staging, and Management of Hepatocellular Carcinoma: 2018 Practice Guidance by the American Association for the Study of Liver Diseases. Hepatology (Baltimore, Md) 68 (2):723-750. doi:10.1002/hep.29913

6. Altekruse S, McGlynn K, Dickie L, Kleiner D (2012) Hepatocellular carcinoma confirmation, treatment, and survival in surveillance, epidemiology, and end results registries, 1992-2008. Hepatology (Baltimore, Md) 55 (2):476-482. doi:10.1002/hep.24710

7. Jo PC, Jang H-J, Burns PN et al. (2017) Integration of Contrast-enhanced US into a Multimodality Approach to Imaging of Nodules in a Cirrhotic Liver: How I Do It. Radiology 282 (2):317-331. doi:10.1148/radiol.2016151732

8. Kono Y, Lyshchik A, Cosgrove D et al. (2017) Contrast Enhanced Ultrasound (CEUS) Liver Imaging Reporting and Data System (LI-RADS $®$ ): the official version by the American College of Radiology (ACR). Ultraschall in der Medizin (Stuttgart, Germany : 1980) 38 (1):85-86. doi:10.1055/s-0042-124369

9. Lyshchik A, Kono Y, Dietrich C et al. (2018) Contrast-enhanced ultrasound of the liver: technical and lexicon recommendations from the ACR CEUS LIRADS working group. Abdominal radiology (New York) 43 (4):861-879. doi:10.1007/s00261-017-1392-0

10. Huang J, Li J, Lu Q et al. (2020) Diagnostic Accuracy of CEUS LI-RADS for the Characterization of Liver Nodules 20 mm or Smaller in Patients at Risk for Hepatocellular Carcinoma. Radiology 294 (2):329-339. doi:10.1148/radiol.2019191086

11. Whiting PF, Rutjes AWS, Westwood ME et al. (2011) QUADAS-2: a revised tool for the quality assessment of diagnostic accuracy studies. Ann Intern Med 155 (8):529-536. doi:10.7326/0003-4819-155-8-201110180-00009

12. Wu XB, Luo HC, Li KY et al. (2018) The application value of LI-RADS classification of contrast enhanced ultrasound in the diagnosis of hepatocellularcarcinoma. Chinese Journal of Ultrasonography 27 (11):936-941. doi:10.3760/cma.j.issn.1004-4477.2018.11.004 (in Chinese)

13. Han H, Kong WT, Qiu YD et al. (2017) Diagnostic value of liver imaging reporting and data system for hepatocellular carcinoma by contrast-enhanced ultrasound. J Clin Ultrasound in Med 19 (08):505-509 (in Chinese)

14. Liu QY (2019) The clinical application value of liver imaging reporting and data system with contrast-enhanced ultrasound in the diagnosis of hepatocellular carcinoma. ZhengZhou University (in Chinese)

15. Zheng W, Li Q, Zou XB et al. (2020) Evaluation of Contrast-enhanced US LI-RADS version 2017: Application on 2020 Liver Nodules in Patients with Hepatitis B Infection. Radiology 294 (2):299-307. doi:10.1148/radiol.2019190878 
16. Chen L, Ruan S, Lin Y et al. (2019) Comparison between M-score and LR-M in the reporting system of contrast-enhanced ultrasound LI-RADS. European radiology 29 (8):4249-4257. doi:10.1007/s00330-018-5927-8

17. Li J, Ling W, Chen S et al. (2019) The interreader agreement and validation of contrast-enhanced ultrasound liver imaging reporting and data system. European journal of radiology 120:108685. doi:10.1016/j.ejrad.2019.108685

18. Schellhaas B, Gortz RS, Pfeifer L et al. (2017) Diagnostic accuracy of contrast-enhanced ultrasound for the differential diagnosis of hepatocellular carcinoma: ESCULAP versus CEUS-LI-RADS. European journal of gastroenterology \& hepatology 29 (9):1036-1044. doi:10.1097/meg.0000000000000916

19. Tan Z, Teoh WC, Wong KM et al. (2020) Analysis of comparative performance of CEUS and CECT/MR LI-RADS classification: Can CEUS dichotomize LIRADS indeterminate lesions on CT or MRI? Clinical imaging 62:63-68. doi:10.1016/j.clinimag.2020.02.002

20. Terzi E, lavarone M, Pompili M et al. (2018) Contrast ultrasound LI-RADS LR-5 identifies hepatocellular carcinoma in cirrhosis in a multicenter restropective study of 1,006 nodules. Journal of hepatology 68 (3):485-492. doi:10.1016/j.jhep.2017.11.007

21. Wang JY, Feng SY, Xu JW et al. (2020) Usefulness of the Contrast-Enhanced Ultrasound Liver Imaging Reporting and Data System in Diagnosing Focal Liver Lesions by Inexperienced Radiologists. Journal of ultrasound in medicine : official journal of the American Institute of Ultrasound in Medicine. doi:10.1002/jum.15242

22. Kang HJ, Kim JH, Joo I, Han JK (2020) Additional value of contrast-enhanced ultrasound (CEUS) on arterial phase non-hyperenhancement observations $(>/=2 \mathrm{~cm}$ ) of CT/MRI for high-risk patients: focusing on the CT/MRI LI-RADS categories LR-3 and LR-4. Abdom Radiol (NY) 45 (1):55-63. doi:10.1007/s00261-019-02132-x

23. CB vdP, CS L, CB S et al. (2019) Accuracy of the Liver Imaging Reporting and Data System in Computed Tomography and Magnetic Resonance Image Analysis of Hepatocellular Carcinoma or Overall Malignancy-A Systematic Review. Gastroenterology 156 (4):976-986. doi:10.1053/j.gastro.2018.11.020

\section{Figures}

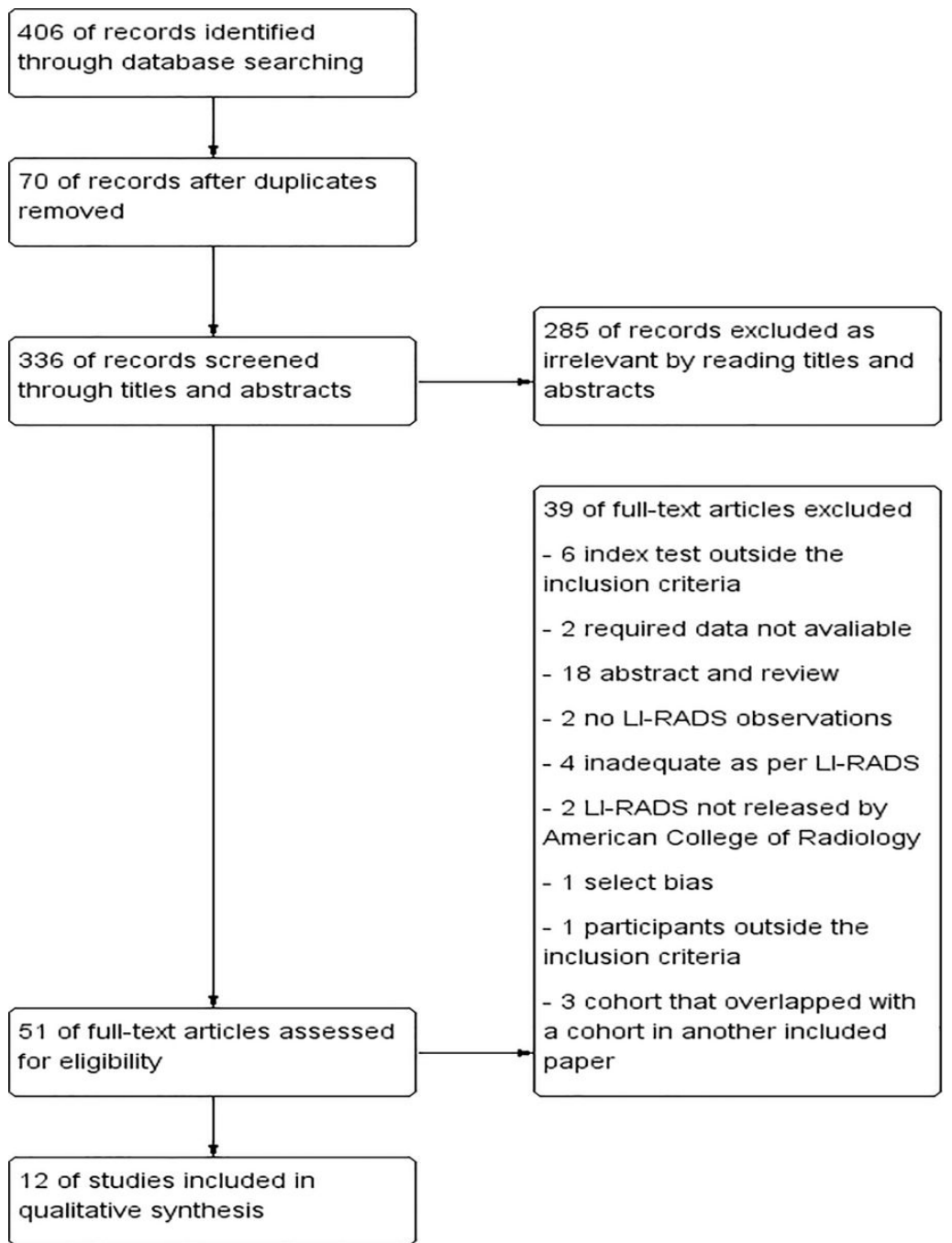

\section{Figure 1}

Study flow diagram. Liver Imaging Reporting and Data System. 

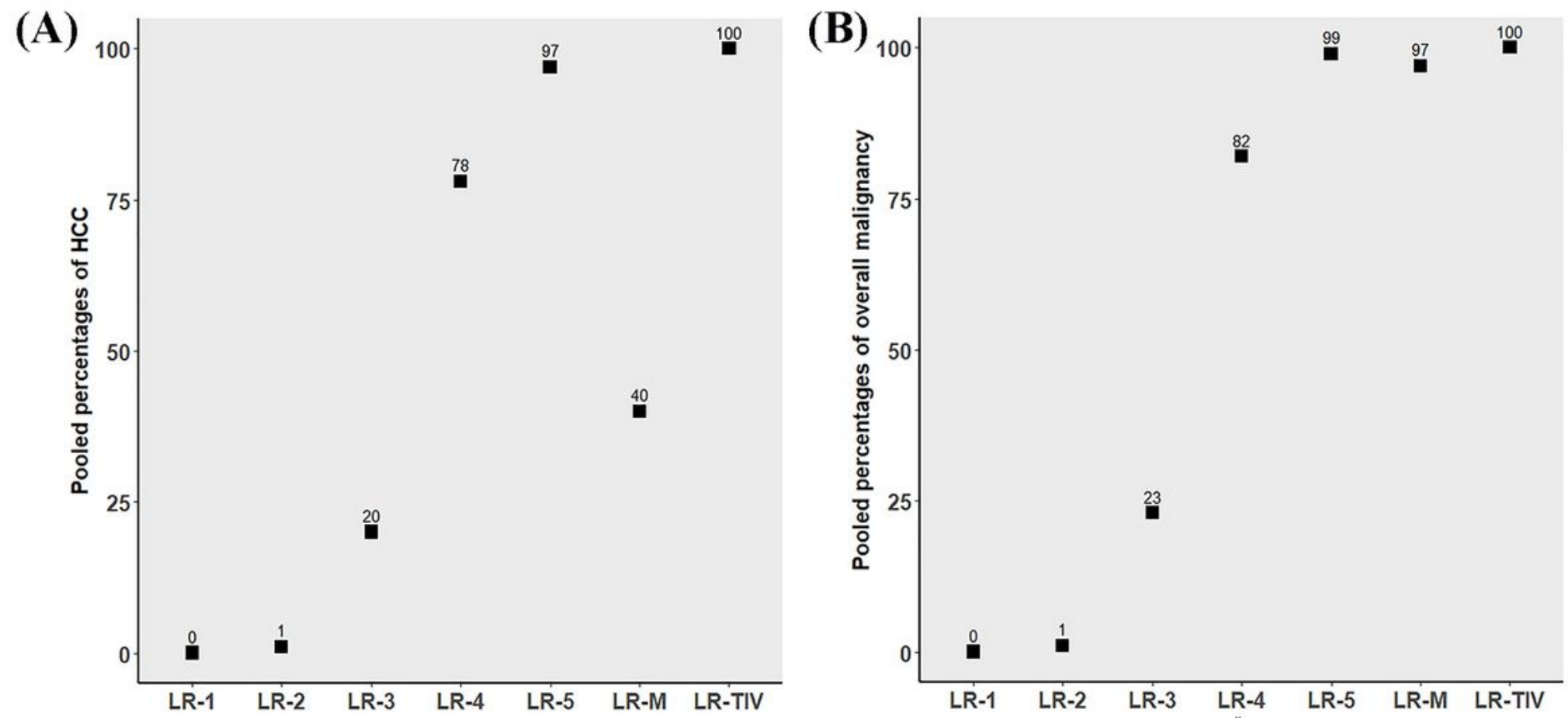

Figure 2

Proportions of HCC (A) and overall malignancy (B) foreach LI-RADS category. Liver Imaging Reporting and Data System; HCC, hepatocellular carcinoma.

\section{Supplementary Files}

This is a list of supplementary files associated with this preprint. Click to download.

- SupplementaryMaterial.docx

- SupplementaryMaterial.docx 